\title{
Le module dendriforme sur le groupe cyclique
}

\author{
F. Chapoton
}

18 août 2021

\begin{abstract}
Résumé
La structure d'opérade anticyclique de l'opérade dendriforme donne en particulier une matrice d'ordre $n$ agissant sur l'espace engendré par les arbres binaires plans à $n$ feuilles. On calcule le polynôme caractéristique de cette matrice. On propose aussi une conjecture compatible pour le polynôme caractéristique de la transformation de Coxeter du poset de Tamari, qui est essentiellement une racine carrée de cette matrice.
\end{abstract}

\begin{abstract}
The structure of anticyclic operad on the Dendriform operad defines in particular a matrix of finite order acting on the vector space spanned by planar binary trees. We compute its characteristic polynomial and propose a (compatible) conjecture for the characteristic polynomial of the Coxeter transformation for the Tamari lattice, which is mostly a square root of this matrix.
\end{abstract}

\section{Introduction}

Les arbres binaires plans sont des objets combinatoires très classiques. Ils ont récemment fait l'objet de recherches remarquables en combinatoire algébrique, suite à leur apparition dans les travaux de Loday [Lod01. Le point de départ est la description par Loday d'une opérade basée sur les arbres binaires plans, nommée opérade dendriforme. A cette opérade correspond une notion d'algèbre dendriforme. Loday et Ronco ont ensuite montré [LR98] que l'algèbre dendriforme libre sur un générateur avait une structure d'algèbre de Hopf. De nombreux travaux [Lod02, LR02, AS06, HNT02, HNT03, HNT05] ont suivi sur différents aspects de ces objets.

L'opérade Dendriforme a en fait une structure plus riche : c'est une opérade anticyclique Cha05a. Ceci donne en particulier une action du groupe cyclique d'ordre $n+1$ sur l'espace vectoriel engendré par les arbres binaires plans à $n$ sommets internes. Cette action reste relativement mystérieuse depuis son introduction, malgré quelques progrès effectués depuis dans sa compréhension. On a montré dans Cha05b] que l'action du générateur $\tau$ du groupe cyclique était liée au carré de la transformation de Coxeter $\theta$ pour le treillis de Tamari. On a aussi montré dans Cha06 que l'action du générateur $\tau$ sur les arbres binaires plans admettait une description très simple par le biais d'un plongement des arbres binaires plans dans les fractions rationnelles.

On calcule ici le polynôme caractéristique du générateur $\tau$ de cette action cyclique. On propose ensuite une conjecture pour le polynôme caractéristique de 
la transformation de Coxeter $\theta$. On montre en particulier que cette conjecture est compatible avec le résultat précédent.

Ce travail a son origine dans une correspondance avec Jean Ecalle, qui a formulé le premier une conjecture sur le polynôme caractéristique de $\tau$.

Merci à Cédric Bonnafé pour la preuve de la Proposition 2.2.

\section{Rappels et notations}

On note Dend l'opérade dendriforme. L'espace vectoriel $\operatorname{Dend}(n)$ a pour base les arbres binaires plans à $n$ sommets internes et pour dimension le nombre de Catalan $\mathbf{c}_{n}$ défini par

$$
\mathbf{c}_{n}=\frac{1}{n+1}\left(\begin{array}{c}
2 n \\
n
\end{array}\right) .
$$

On trouve dans Cha05a la description en termes d'opérade d'une action naturelle du groupe cyclique d'ordre $n$ sur $\operatorname{Dend}(n-1)$. On a donc un module induit sur le groupe symétrique $\mathfrak{S}_{n}$.

Proposition 1.1 ([Cha05a], Th. 6.2) Le caractère de ce module induit est donné par la fonction symétrique

$$
2 \mathbf{c}_{n-1} p_{1}^{n}-\frac{1}{2 n} \sum_{d \mid n} \phi(d)\left(\begin{array}{c}
2 n / d \\
n / d
\end{array}\right) p_{d}^{n / d},
$$

où $\phi$ est l'indicatrice d'Euler et les $p_{d}$ sont les fonctions symétriques "sommes de puissances".

Cette fonction symétrique décrit donc le module sur le groupe symétrique $\mathfrak{S}_{n}$ induit depuis un module Dend $(n-1)$ sur le groupe cyclique $C_{n}$. On cherche à comprendre ce module sur le groupe cyclique, dont on sait a priori qu'il est défini sur les rationnels et même sur les entiers.

On voit que la formule (21) se décompose en une somme de deux termes. Le premier terme $2 \mathbf{c}_{n-1} p_{1}^{n}$ est une somme de $2 \mathbf{c}_{n-1}$ représentations régulières de $\mathfrak{S}_{n}$, donc est isomorphe à l'induite de la somme de $2 \mathbf{c}_{n-1}$ représentations régulières de $C_{n}$. On se concentre donc par la suite sur le deuxième terme.

\section{Induction du groupe cyclique au groupe symétrique}

Soit $C_{n}$ le groupe cyclique à $n$ éléments. On note $t$ le générateur. L'algèbre de groupe est $\mathbb{Q}[t] /\left(t^{n}-1\right)$. $M_{n, d}$.

Soit $d$ un diviseur de $n$. L'espace $\mathbb{Q}[t] /\left(t^{d}-1\right)$ est un module pour $C_{n}$, noté

Remarque : $M_{n, n}$ est la représentation régulière.

Le caractère de $M_{n, d}$ est le suivant :

$$
\chi_{n, d}\left(t^{k}\right)=\left\{\begin{array}{l}
d \text { si } d \mid k, \\
0 \text { sinon. }
\end{array}\right.
$$

Soit $K_{0}^{\mathbb{Q}}\left(C_{n}\right)$ le groupe de Grothendieck de la catégorie des $\mathbb{Q} C_{n}$-modules de type fini. Le groupe $K_{0}^{\mathbb{Q}}\left(C_{n}\right)$ a pour rang le nombre de diviseurs de $n$ et les 
classes dans $K_{0}^{\mathbb{Q}}\left(C_{n}\right)$ des modules $M_{n, d}$ pour $d \mid n$ forment une base de $K_{0}^{\mathbb{Q}}\left(C_{n}\right)$ CR62, §39, Ex. 2].

Soit $\mathfrak{S}_{n}$ le groupe symétrique sur $\{1, \ldots, n\}$.

On a une inclusion de groupe de $C_{n}$ dans $\mathfrak{S}_{n}$ qui envoie $t$ sur le grand cycle $c=(1,2, \ldots, n)$.

On considère le module induit $M_{n, d}^{\prime}=\operatorname{Ind}_{C_{n}}^{\mathfrak{S}_{n}} M_{n, d}$.

Proposition 2.1 Le caractère de $M_{n, d}^{\prime}$ est la fonction symétrique

$$
\chi_{n, d}^{\prime}=\frac{d}{n} \sum_{\ell \mid n / d} \phi(\ell) p_{\ell}^{n / \ell} .
$$

Preuve. Par la formule d'induction des caractères, le caractère $\chi_{n, d}^{\prime}$ est donné (en tant que fonction centrale sur le groupe symétrique) par

$$
\chi_{n, d}^{\prime}(\sigma)=\frac{1}{n} \sum_{\substack{\tau \in \mathfrak{N}_{n} \\ \tau-1 \\ \tau \sigma \in C_{n}}} \chi_{n, d}\left(\tau^{-1} \sigma \tau\right),
$$

pour toute permutation $\sigma$. En utilisant (3), ceci se simplifie en

$$
\frac{d}{n} \sum_{d \mid k} \sum_{\substack{\tau \in \mathfrak{S}_{n} \\ \tau-1 \\ \sigma \tau=c^{k}}} 1 .
$$

On note que le type cyclique de $c^{k}$ est $\left(\frac{n}{n \wedge k}\right)^{n \wedge k}$. On traduit alors le caractère $\chi_{n, d}^{\prime}$ en termes de fonctions symétriques:

$$
\chi_{n, d}^{\prime}=\frac{d}{n} \sum_{\substack{k=1 \ldots n \\ d \mid k}}\left(p_{\frac{n}{n \wedge k}}\right)^{n \wedge k} .
$$

On remplace ensuite $k$ par $d k$ et $n$ par $d m$ :

$$
\frac{d}{n} \sum_{k=1}^{m}\left(p_{\frac{m}{m \wedge k}}\right)^{(m \wedge k) d}
$$

On trouve la formule attendue en regroupant les termes.

Proposition 2.2 L'induction $\operatorname{Ind}_{C_{n}}^{\mathfrak{S}_{n}}$ de $K_{0}^{\mathbb{Q}}\left(C_{n}\right)$ dans $K_{0}^{\mathbb{Q}}\left(\mathfrak{S}_{n}\right)$ est une application linéaire injective.

Preuve. Comme les classes des modules $M_{n, d}$ forment une base de $K_{0}^{\mathbb{Q}}\left(C_{n}\right)$, il suffit de montrer que les classes des modules induits $M_{n, d}^{\prime}$ dans $K_{0}^{\mathbb{Q}}\left(\mathfrak{S}_{n}\right)$ sont linéairement indépendantes. Ceci résulte immédiatement de la formule (4), par triangularité par rapport à l'ordre partiel défini sur l'ensemble des diviseurs de $n$ par la divisibilité.

\section{Description du module dendriforme}

On définit une suite d'entiers positifs $\mathbf{a}_{n}$ pour $n \geq 1$ par la formule suivante :

$$
\mathbf{a}_{n}=\frac{1}{2 n} \sum_{d \mid n} \mu(n / d)\left(\begin{array}{c}
2 d \\
d
\end{array}\right)
$$


où $\mu$ est la fonction de Möbius.

A priori, les $\mathbf{a}_{n}$ définis ainsi sont des rationnels. Il faut justifier que ce sont bien des entiers positifs. Pour cela, on va faire un calcul plus fin avec des fonctions symétriques. On note $\circ$ le pléthysme des fonctions symétriques.

Considérons la fonction symétrique Lie :

$$
\text { Lie }=\sum_{n \geq 1} \frac{1}{n} \sum_{d \mid n} \mu(d) p_{d}^{n / d} .
$$

C'est la fonction symétrique associée à l'opérade Lie, donc c'est une somme positive de fonctions de Schur.

Considérons la fonction symétrique Brace :

$$
\text { Brace }=\sum_{n \geq 1} \frac{1}{n}\left(\begin{array}{c}
2 n-2 \\
n-1
\end{array}\right) p_{1}^{n}=\frac{1-\sqrt{1-4 p_{1}}}{2} .
$$

C'est aussi clairement une somme positive de fonctions de Schur.

Proposition 3.1 Le pléthysme Lie o Brace est la fonction symétrique

$$
\sum_{n \geq 1} \frac{1}{2 n} \sum_{d \mid n} \mu(d)\left(\begin{array}{c}
2 n / d \\
n / d
\end{array}\right) p_{d}^{n / d}
$$

Preuve. Par définition du pléthysme, il faut calculer

$$
\sum_{n \geq 1} \frac{1}{n} \sum_{d \mid n} \mu(d)\left(\frac{1-\sqrt{1-4 p_{d}}}{2}\right)^{n / d} .
$$

En échangeant les sommations, ceci devient

$$
-\sum_{d \geq 1} \frac{\mu(d)}{d} \log \left(\frac{1+\sqrt{1-4 p_{d}}}{2}\right) .
$$

En utilisant alors le développement de Taylor (72) (Voir Appendice) :

$$
-\log \left(\frac{1+\sqrt{1-4 u}}{2}\right)=\sum_{n \geq 1} \frac{1}{2 n}\left(\begin{array}{c}
2 n \\
n
\end{array}\right) u^{n},
$$

on obtient donc

$$
\sum_{d \geq 1} \frac{\mu(d)}{d} \sum_{k \geq 1} \frac{1}{2 k}\left(\begin{array}{c}
2 k \\
k
\end{array}\right) p_{d}^{k}
$$

qui se réduit facilement au résultat voulu.

Comme composée pour le pléthysme de sommes positives de fonctions de Schur, la fonction Lie o Brace est aussi une somme positive de fonctions de Schur. La dimension des invariants dans Lie o Brace, obtenue en posant $p_{d}=1$ pour tout $d$ dans les composantes homogènes de la formule (12), est exactement la suite $\mathbf{a}_{n}$; ces nombres sont donc bien des entiers positifs.

Voici les premiers termes de la suite $\mathbf{a}_{n}$ pour $n \geq 1$ :

$$
1,1,3,8,25,75,245,800,2700,9225, \ldots
$$

La suite $\mathbf{a}_{n}$ a une propriété remarquable. 
Proposition 3.2 On a

$$
F_{\mathbf{a}}(x)=\prod_{n \geq 1}\left(1-x^{n}\right)^{-\mathbf{a}_{n}}=\frac{1-\sqrt{1-4 x}}{2 x} .
$$

Preuve. Soit Com la fonction symétrique associée à l'opérade Com et correspondant à la somme des modules triviaux sur les groupes symétriques. Elle vérifie $(1+\mathrm{Com}) \circ \mathrm{Lie}=\frac{1}{1-p_{1}}$. Par conséquent, on a

$$
(1+\text { Com }) \circ(\text { Lie } \circ \text { Brace })=\left(\frac{1}{1-p_{1}}\right) \circ \text { Brace } .
$$

En regardant les invariants de part et d'autre, on en déduit la proposition.

On utilise maintenant cette suite d'entiers $\mathbf{a}_{n}$ pour décrire le module dendriforme Dend $(n-1)$ sur le groupe cyclique $C_{n}$.

Proposition 3.3 Pour tout $n \geq 1$, on a l'égalité suivante:

$$
\sum_{d \mid n} \mathbf{a}_{d} \chi_{n, d}^{\prime}=\frac{1}{2 n} \sum_{d \mid n} \phi(d)\left(\begin{array}{c}
2 n / d \\
n / d
\end{array}\right) p_{d}^{n / d} .
$$

Preuve. On calcule la somme pour $n \geq 1 \mathrm{du}$ membre de gauche. En utilisant l'expression (4) de $\chi_{n, d}^{\prime}$, on obtient

$$
\sum_{n \geq 1} \sum_{d \mid n} \frac{1}{2 d} \sum_{k \mid d} \mu(d / k)\left(\begin{array}{c}
2 k \\
k
\end{array}\right) \frac{d}{n} \sum_{\ell \mid n / d} \phi(\ell) p_{\ell}^{n / \ell}
$$

ce qui s'écrit encore

$$
\sum_{n \geq 1} \sum_{d \mid n} \sum_{k \mid d} \sum_{\ell \mid n / d} \frac{1}{2 n} \mu(d / k)\left(\begin{array}{c}
2 k \\
k
\end{array}\right) \phi(\ell) p_{\ell}^{n / \ell} .
$$

On introduit de nouvelles variables de sommation $i$ et $j$ en posant $n=i j k \ell$ et $d=i k$. En remplaçant les sommations sur $n$ et $d$ par des sommations sur $i$ et $j$, on obtient

$$
\sum_{i, j, k, \ell} \frac{1}{2 i j k \ell} \mu(i) \phi(\ell)\left(\begin{array}{c}
2 k \\
k
\end{array}\right) p_{\ell}^{i j k} .
$$

En utilisant le pléthysme des fonctions symétriques, on peut factoriser cette expression comme suit :

$$
\left(\sum_{i} \mu(i) p_{i} / i\right) \circ\left(\sum_{j} p_{j} / j\right) \circ\left(\sum_{k, \ell} \frac{1}{2 k \ell} \phi(\ell)\left(\begin{array}{c}
2 k \\
k
\end{array}\right) p_{\ell}^{k}\right) .
$$

Comme les deux premiers termes sont dans un sous-groupe des fonctions symétriques pour le pléthysme qui est isomorphe au groupe commutatif des séries de Dirichlet pour le produit, ces termes sont inverses l'un de l'autre. On obtient donc

$$
\sum_{k, \ell} \frac{1}{2 k \ell} \phi(\ell)\left(\begin{array}{c}
2 k \\
k
\end{array}\right) p_{\ell}^{k} .
$$

Il est facile de voir que le terme de degré $n$ de cette somme est exactement le résultat attendu. 
On a donc montré que l'induite du module virtuel

$$
2 \mathbf{c}_{n-1} M_{n, n}-\bigoplus_{d \mid n} \mathbf{a}_{d} M_{n, d}
$$

a le même caractère que l'induite du module Dend $(n-1)$.

On sait aussi que le module $\operatorname{Dend}(n-1)$ est défini sur les rationnels et même sur les entiers. On sait par ailleurs que les modules $M_{n, d}$ sont aussi définis sur les rationnels.

Par la proposition 2.2, on en déduit

Theorem 3.4 Le module $\operatorname{Dend}(n-1)$ a pour caractère

$$
2 \mathbf{c}_{n-1} \chi_{n, n}-\bigoplus_{d \mid n} \mathbf{a}_{d} \chi_{n, d}
$$

et le polynôme caractéristique du générateur $t$ de $C_{n}$ est

$$
\frac{\left(t^{n}-1\right)^{2 \mathbf{c}_{n-1}}}{\prod_{d \mid n}\left(t^{d}-1\right)^{\mathbf{a}_{d}}} \text {. }
$$

Ce théorème incite à penser que le module $\operatorname{Dend}(n-1)$ doit admettre une résolution de la forme

$$
0 \longrightarrow \bigoplus_{d \mid n} \mathbf{a}_{d} M_{n, d} \longrightarrow 2 \mathbf{c}_{n-1} M_{n, n} \longrightarrow \operatorname{Dend}(n-1) \longrightarrow 0 .
$$

On a une application évidente de $c_{n-1} M_{n, n}$ dans Dend $(n-1)$, induite par l'identité de Dend $(n-1)$. Pour définir un morphisme de $2 c_{n-1} M_{n, n}$ dans $\operatorname{Dend}(n-1)$, il faudrait une autre application de $c_{n-1} M_{n, n}$ dans $\operatorname{Dend}(n-1)$. On peut supposer qu'elle doit provenir d'une involution sur Dend $(n-1)$.

Remarque : dans le cas similaire mais plus simple de l'opérade Dias, dont le caractère anticyclique est donné par $M_{n, n}^{\prime}-M_{n, 1}^{\prime}$, on retrouve la description connue du module $\operatorname{Dias}(n-1)$ comme quotient de $M_{n, n}$ par $M_{n, 1}$. On a une suite exacte courte

$$
0 \longrightarrow M_{n, 1} \longrightarrow M_{n, n} \longrightarrow \operatorname{Dias}(n-1) \longrightarrow 0 \text {. }
$$

\section{Transformation de Coxeter}

Le treillis de Tamari HT72] est un ordre partiel sur les arbres binaires plans à $n$ feuilles. Soit $C$ la matrice de cet ordre :

$$
C_{x, y}=\left\{\begin{array}{l}
1 \text { si } x \leq y \\
0 \text { sinon }
\end{array}\right.
$$

La matrice $\theta=-C\left({ }^{t} C^{-1}\right)$ est appelée la transformation de Coxeter du poset de Tamari.

On a montré dans Cha05b la relation suivante. 
Proposition 4.1 ([Cha05b], Th. 6.1) Si $\tau$ est la matrice d'ordre n considérée précédemment et $\theta$ la transformation de Coxeter du treillis de Tamari, alors on $a$

$$
\tau=(-1)^{n+1} \theta^{2} .
$$

Il est donc naturel de se demander si il existe une description simple du polynôme caractéristique de $\theta$. On propose ci-dessous une conjecture pour ce polynôme. Il est nécessaire de distinguer les cas $n$ pair et $n$ impair.

On commence par introduire une suite d'entiers relatifs $\lambda(n)$.

$$
\lambda(n)=(-1)^{\left(\begin{array}{c}
n \\
2
\end{array}\right)}\left(\begin{array}{c}
n-1 \\
\lceil(n-1) / 2\rceil
\end{array}\right) .
$$

On définit ensuite une suite d'entiers relatifs $\mathbf{b}_{n}$ par inversion de Möbius :

$$
\mathbf{b}_{n}=\frac{1}{n} \sum_{d \mid n} \mu(d) \lambda(n / d) .
$$

A priori, les $\mathbf{b}_{n}$ sont des rationnels. Il faut justifier que ce sont bien des entiers relatifs. On procède comme pour la suite $\mathbf{a}_{n}$, en utilisant des fonctions symétriques.

On introduit la fonction symétrique

$$
Z=p_{1}+\sum_{n \geq 0} \frac{(-1)^{n+1}}{n+1}\left(\begin{array}{c}
2 n \\
n
\end{array}\right) p_{1}^{2 n+2}=\frac{1+2 p_{1}-\sqrt{1+4 p_{1}^{2}}}{2} .
$$

Proposition 4.2 La fonction symétrique Lie $\circ Z$ est

$$
\sum_{n \geq 1} \frac{1}{n} \sum_{d \mid n} \mu(d) \lambda(n / d) p_{d}^{n / d} .
$$

Preuve. La preuve est similaire à celle de la Prop. 3.1 Par définition du pléthysme, il faut calculer

$$
\sum_{n \geq 1} \frac{1}{n} \sum_{d \mid n} \mu(d)\left(\frac{1+2 p_{d}-\sqrt{1+4 p_{d}^{2}}}{2}\right)^{n / d} .
$$

En échangeant les sommations, on obtient

$$
-\sum_{d \geq 1} \frac{\mu(d)}{d} \log \left(\frac{1-2 p_{d}+\sqrt{1+4 p_{d}^{2}}}{2}\right) .
$$

On utilise alors le développement de Taylor (76) (Voir Appendice) :

$$
-\log \left(\frac{1-2 u+\sqrt{1+4 u^{2}}}{2}\right)=\sum_{n \geq 1} \lambda(n) u^{n} / n .
$$

On obtient donc

$$
\sum_{d \geq 1} \frac{\mu(d)}{d} \sum_{k \geq 1} \frac{\lambda(k)}{k} p_{d}^{k}
$$

qui se réduit facilement au résultat voulu. 
Comme Lie et $Z$ sont des combinaisons linéaires entières de fonctions de Schur, la fonction symétrique Lie $\circ Z$ l'est aussi. Ceci montre que les $\mathbf{b}_{n}$ sont bien des entiers relatifs, car ce sont les coefficients des invariants dans cette fonction symétrique.

Voici les premiers termes de la suite $\mathbf{b}_{n}$ pour $n \geq 1$ :

$$
1,-1,-1,1,1,-1,-3,4,8,-13,-23,39,71,-121, \ldots
$$

On remarque que les signes des $\mathbf{b}_{n}$ semblent suivre un motif régulier, le même que pour la suite $\lambda(n)$. En fait, il semble même que la fonction symétrique Lie $\circ Z$ soit une somme de fonctions de Schur dont les signes sont soit tous positifs, soit tous négatifs selon la valeur de $n$ modulo 4 . On a vérifié ceci pour $n \leq 12$.

\section{Proposition 4.3 On a}

$$
F_{\mathbf{b}}(x)=\prod_{n \geq 1}\left(1-x^{n}\right)^{-\mathbf{b}_{n}}=\frac{-1+2 x+\sqrt{1+4 x^{2}}}{2 x} .
$$

Preuve. On utilise l'identité

$$
(1+\mathrm{Com}) \circ(\operatorname{Lie} \circ Z)=\frac{1}{1-p_{1}} \circ Z .
$$

En prenant les invariants, on trouve l'égalité voulue.

On utilise maintenant la suite de nombres entiers relatifs $\mathbf{b}_{n}$ pour proposer une description de $\theta$.

Conjecture 4.4 Pour n pair, le polynôme caractéristique de $\theta$ est

$$
\frac{\left(t^{2 n}-1\right)^{\mathbf{c}_{n-1}}}{\prod_{d \mid 2 n}\left(t^{d}-1\right)^{\mathbf{b}_{d}}} .
$$

Pour donner une conjecture dans le cas $n$ impair, on définit une autre suite d'entiers relatifs $\mathbf{b}_{n}^{\prime}$ à partir de la suite $\mathbf{b}_{n}$ :

$$
\mathbf{b}_{n}^{\prime}= \begin{cases}\mathbf{b}_{n} & \text { si } n=1 \bmod 2 \\ -\mathbf{b}_{n}-\mathbf{b}_{n / 2} & \text { si } n=0 \bmod 2 .\end{cases}
$$

Voici les premiers termes de la suite $\mathbf{b}_{n}^{\prime}$ pour $n \geq 1$ :

$$
1,0,-1,0,1,2,-3,-5,8,12,-23,-38,71,124, \ldots
$$

Conjecture 4.5 Pour n impair, le polynôme caractéristique de $\theta$ est

$$
\frac{\left(t^{2 n}-1\right)^{\mathbf{c}_{n-1}}}{\prod_{d \mid 2 n}\left(t^{d}-1\right)^{\mathbf{b}_{d}^{\prime}}}
$$




\section{Comparaison entre conjectures et théorème}

On montre ici que les conjectures 4.4 et 4.5 sont compatibles avec (et impliquent) le théorème 3.4 décrivant le polynôme caractéristique pour $\tau$.

Le polynôme caractéristique du carré d'une matrice $M$ d'ordre fini est obtenu par la substitution suivante dans celui de $M$ :

$$
\left(t^{d}-1\right) \mapsto \begin{cases}t^{d}-1 & \text { si } d=1 \bmod 2, \\ \left(t^{d / 2}-1\right)^{2} & \text { si } d=0 \bmod 2 .\end{cases}
$$

Le polynôme caractéristique de l'opposé d'une matrice $M$ d'ordre fini est obtenu par la substitution suivante dans celui de $M$ :

$$
\left(t^{d}-1\right) \mapsto \begin{cases}\left(t^{2 d}-1\right) /\left(t^{d}-1\right) & \text { si } d=1 \bmod 2 \\ \left(t^{d}-1\right) & \text { si } d=0 \bmod 2 .\end{cases}
$$

Supposons d'abord $n$ impair et considérons la conjecture 4.5. Dans ce cas, on a $\tau=\theta^{2}$. On obtient donc l'expression suivante pour le polynôme caractéristique de $\tau$ :

$$
\left(t^{n}-1\right)^{2 \mathbf{c}_{n-1}} \prod_{\substack{d \mid 2 n \\ d=1 \bmod 2}}\left(t^{d}-1\right)^{-\mathbf{b}_{d}^{\prime}} \prod_{\substack{d \mid 2 n \\ d=0 \bmod 2}}\left(t^{d / 2}-1\right)^{-2 \mathbf{b}_{d}^{\prime}} .
$$

Ceci se ré-écrit

$$
\left(t^{n}-1\right)^{2 \mathbf{c}_{n-1}} \prod_{d \mid n}\left(t^{d}-1\right)^{-\mathbf{b}_{d}^{\prime}-2 \mathbf{b}_{2 d}^{\prime}} .
$$

Pour identifier ceci à la formule (28), il faut donc avoir

$$
\mathbf{a}_{d}=\mathbf{b}_{d}^{\prime}+2 \mathbf{b}_{2 d}^{\prime}
$$

pour tous les entiers $d$ impairs. En utilisant les relations (45), ceci est équivalent à

$$
\mathbf{a}_{d}=-\mathbf{b}_{d}-2 \mathbf{b}_{2 d}
$$

pour tous les entiers $d$ impairs.

Supposons maintenant $n$ pair et considérons la conjecture 4.4 Dans ce cas, on a $\tau=-\theta^{2}$. On obtient donc l'expression suivante pour le polynôme caractéristique de $\tau$ :

$\left(t^{n}-1\right)^{2 \mathbf{c}_{n-1}} \prod_{\substack{d \mid n \\ d=0 \bmod 2}}\left(t^{d}-1\right)^{-2 \mathbf{b}_{2 d}} \prod_{\substack{d \mid n \\ d=1 \bmod 2}} \frac{\left(t^{2 d}-1\right)^{-2 \mathbf{b}_{2 d}}}{\left(t^{d}-1\right)^{-2 \mathbf{b}_{2 d}}} \prod_{\substack{d \mid n \\ d=1 \bmod 2}} \frac{\left(t^{2 d}-1\right)^{-\mathbf{b}_{d}}}{\left(t^{d}-1\right)^{-\mathbf{b}_{d}}}$.

Pour identifier cette expression avec la formule (28), on distingue selon la valeur de $d$ modulo 4 . On obtient les conditions suivantes :

$$
\mathbf{a}_{d}= \begin{cases}-2 \mathbf{b}_{2 d}-\mathbf{b}_{d} & \text { si } d=1 \bmod 2 \\ 2 \mathbf{b}_{2 d} & \text { si } d=0 \bmod 4 \\ 2 \mathbf{b}_{2 d}+2 \mathbf{b}_{d}+\mathbf{b}_{d / 2} & \text { si } d=2 \bmod 4\end{cases}
$$

On remarque que la condition (53) obtenue plus haut dans le cas $n$ impair fait aussi partie des trois conditions ci-dessus. 
Proposition 5.1 On a la relation

$$
\begin{aligned}
& \prod_{n}\left(1-x^{n}\right)^{-\mathbf{a}_{n}}= \\
& \prod_{n=0 \bmod 4}\left(1-x^{n}\right)^{-2 \mathbf{b}_{2 n}} \prod_{n=2 \bmod 4}\left(1-x^{n}\right)^{-2 \mathbf{b}_{2 n}-2 \mathbf{b}_{n}-\mathbf{b}_{n / 2}} \prod_{n=1 \bmod 2}\left(1-x^{n}\right)^{2 \mathbf{b}_{2 n}+\mathbf{b}_{n}} .
\end{aligned}
$$

Preuve. Calculons le second membre :

$$
\prod_{n=0 \bmod 2}\left(1-x^{2 n}\right)^{-2 \mathbf{b}_{4 n}} \prod_{n=1 \bmod 2}\left(1-x^{2 n}\right)^{-2 \mathbf{b}_{4 n}-2 \mathbf{b}_{2 n}-\mathbf{b}_{n}} \prod_{n=1 \bmod 2}\left(1-x^{n}\right)^{2 \mathbf{b}_{2 n}+\mathbf{b}_{n}} .
$$

On coupe en deux le facteur central et on regroupe :

$$
\prod_{n}\left(1-x^{2 n}\right)^{-2 \mathbf{b}_{4 n}} \prod_{n=1 \bmod 2}\left(1+x^{n}\right)^{-2 \mathbf{b}_{2 n}-\mathbf{b}_{n}} .
$$

On ré-écrit le premier facteur et on coupe en deux le second :

$$
\prod_{n=0 \bmod 2}\left(1-x^{n}\right)^{-2 \mathbf{b}_{2 n}} \prod_{n=1 \bmod 2}\left(1+x^{n}\right)^{-2 \mathbf{b}_{2 n}} \prod_{n=1 \bmod 2}\left(1+x^{n}\right)^{-\mathbf{b}_{n}} .
$$

On regroupe les deux premiers facteurs et on ré-écrit le troisième :

$$
\prod_{n}\left(1-(-x)^{n}\right)^{-2 \mathbf{b}_{2 n}} \prod_{n=1 \bmod 2}\left(1-(-x)^{n}\right)^{-\mathbf{b}_{n}} .
$$

On complète le second facteur :

$$
\prod_{n}\left(1-(-x)^{n}\right)^{-2 \mathbf{b}_{2 n}} \prod_{n}\left(1-(-x)^{2 n}\right)^{\mathbf{b}_{2 n}} \prod_{n}\left(1-(-x)^{n}\right)^{-\mathbf{b}_{n}} .
$$

Le troisième facteur est $F_{\mathbf{b}}(-x)$, la fonction $F_{\mathbf{b}}$ étant définie par (42). On regroupe les deux premiers facteurs et on simplifie :

$$
\prod_{n}\left(\frac{1-(-x)^{n}}{1+(-x)^{n}}\right)^{-\mathbf{b}_{2 n}} F_{\mathbf{b}}(-x) .
$$

On pose $x=-z^{2}$ et on choisit les signes avec soin :

$$
\prod_{n}\left(\frac{1-z^{2 n}}{1+(-z)^{2 n}}\right)^{-\mathbf{b}_{2 n}} F_{\mathbf{b}}\left(z^{2}\right)
$$

soit encore

$$
\prod_{n=0 \text { mod } 2}\left(\frac{1-z^{n}}{1+(-z)^{n}}\right)^{-\mathbf{b}_{n}} F_{\mathbf{b}}\left(z^{2}\right)
$$

On peut alors compléter le produit sans introduire de nouveaux termes :

$$
\prod_{n}\left(\frac{1-z^{n}}{1+(-z)^{n}}\right)^{-\mathbf{b}_{n}} F_{\mathbf{b}}\left(z^{2}\right)
$$


soit enfin

$$
F_{\mathbf{b}}(z) \prod_{n}\left(1-(-z)^{2 n}\right)^{\mathbf{b}_{n}} \prod_{n}\left(1-(-z)^{n}\right)^{-\mathbf{b}_{n}} F_{\mathbf{b}}\left(z^{2}\right) .
$$

On obtient donc

$$
F_{\mathbf{b}}(z)\left(1 / F_{\mathbf{b}}\left(z^{2}\right)\right) F_{\mathbf{b}}(-z) F_{\mathbf{b}}\left(z^{2}\right)=F_{\mathbf{b}}(z) F_{\mathbf{b}}(-z),
$$

ce qui vaut bien

$$
\frac{1-\sqrt{1+4 z^{2}}}{-2 z^{2}}=\frac{1-\sqrt{1-4 x}}{2 x} .
$$

Ceci est bien la fonction $F_{\mathbf{a}}(x)$ définie en (18), comme attendu.

Remarque : on a montré au passage la relation

$$
F_{\mathbf{a}}\left(-z^{2}\right)=F_{\mathbf{b}}(z) F_{\mathbf{b}}(-z) .
$$

\section{Appendice}

On rappelle quelques développements de Taylor.

Le premier est classique et facile :

$$
\sum_{n \geq 0}\left(\begin{array}{c}
2 n \\
n
\end{array}\right) x^{n}=\frac{1}{\sqrt{1-4 x}}
$$

On déduit de (70) en utilisant l'opérateur $x \partial_{x}$ :

$$
\sum_{n \geq 1} \frac{1}{n}\left(\begin{array}{c}
2 n-2 \\
n-1
\end{array}\right) x^{n}=\frac{1-\sqrt{1-4 x}}{2} .
$$

On déduit aussi de (70) en utilisant l'opérateur $x \partial_{x}$ :

$$
\sum_{n \geq 1} \frac{1}{2 n}\left(\begin{array}{c}
2 n \\
n
\end{array}\right) x^{n}=-\log \left(\frac{1+\sqrt{1-4 x}}{2}\right) .
$$

On déduit de (71) en remplaçant $x$ par $-x^{2}$ :

$$
\sum_{n \geq 0} \frac{(-1)^{n+1}}{n+1}\left(\begin{array}{c}
2 n \\
n
\end{array}\right) x^{2 n+1}=\frac{1-\sqrt{1+4 x^{2}}}{2 x} .
$$

On démontre ensuite

$$
\sum_{n \geq 1} \lambda_{n} x^{n}=\frac{x}{\sqrt{1+4 x^{2}}}-\frac{1}{2}\left(1-\frac{1}{\sqrt{1+4 x^{2}}}\right),
$$

en séparant les puissances paires et impaires de $x$ et en utilisant la relation

$$
\left(\begin{array}{c}
2 n \\
n
\end{array}\right)=2\left(\begin{array}{c}
2 n-1 \\
n
\end{array}\right)
$$

On déduit ensuite de (174) en utilisant l'opérateur $x \partial_{x}$ :

$$
\sum_{n \geq 1} \frac{\lambda_{n}}{n} x^{n}=-\log \left(\frac{1-2 x+\sqrt{1+4 x^{2}}}{2}\right) .
$$




\section{Références}

[AS06] M. Aguiar and F. Sottile. Structure of the Loday-Ronco Hopf algebra of trees. J. Algebra, 295(2) :473-511, 2006.

[Cha05a] F. Chapoton. On some anticyclic operads. Algebr. Geom. Topol., 5 :53-69 (electronic), 2005.

[Cha05b] F. Chapoton. On the Coxeter transformations for Tamari posets, 2005. arXiv :math.QA/0502065, to appear in Bull. Canad. Math.

[Cha06] F. Chapoton. The anticyclic operad of moulds. math.QA/0609436, 2006.

[CR62] C. W. Curtis and I. Reiner. Representation theory of finite groups and associative algebras. Pure and Applied Mathematics, Vol. XI. Interscience Publishers, a division of John Wiley \& Sons, New YorkLondon, 1962.

[HNT02] F. Hivert, J.-C. Novelli, and J.-Y. Thibon. Un analogue du monoïde plaxique pour les arbres binaires de recherche. C. R. Math. Acad. Sci. Paris, 335(7) :577-580, 2002.

[HNT03] F. Hivert, J.-C. Novelli, and J.-Y. Thibon. Sur quelques propriétés de l'algèbre des arbres binaires. C. R. Math. Acad. Sci. Paris, 337(9) :565-568, 2003.

[HNT05] F. Hivert, J.-C. Novelli, and J.-Y. Thibon. The algebra of binary search trees. Theoret. Comput. Sci., 339(1) :129-165, 2005.

[HT72] S. Huang and D. Tamari. Problems of associativity : A simple proof for the lattice property of systems ordered by a semi-associative law. J. Combinatorial Theory Ser. A, 13 :7-13, 1972.

[Lod01] J.-L. Loday. Dialgebras. In Dialgebras and related operads, volume 1763 of Lecture Notes in Math., pages 7-66. Springer, Berlin, 2001.

[Lod02] J.-L. Loday. Arithmetree. J. Algebra, 258(1):275-309, 2002. Special issue in celebration of Claudio Procesi's 60th birthday.

[LR98] J.-L. Loday and M. O. Ronco. Hopf algebra of the planar binary trees. Adv. Math., 139(2):293-309, 1998.

[LR02] J.-L. Loday and M. O. Ronco. Order structure on the algebra of permutations and of planar binary trees. J. Algebraic Combin., $15(3): 253-270,2002$. 